\title{
Antimicrobial investigation on the multi-state outbreak of salmonellosis and shigellosis in Iran
}

\author{
Mohammad Mehdi Soltan Dallal*1,2(D), Samaneh Motalebi², Hossein Masoumi Asl ${ }^{3}$, Mohammad Kazem Sharifi Yazdi,5, \\ Abbas Rahimi Forushani ${ }^{6}$
}

Received: 28 Jan 2018

Published: 18 May 2020

\section{Abstract}

Background: Foodborne diseases are caused by indigestion of contaminated food. In some cases they may result in either hospitalization or death. The Centers for Disease Control (CDC) and Prevention in 2017 stated that 10\% reduction in foodborne illness would prevent nearly five million illnesses every year. Approximately one out of six Americans become ill from contaminated foods or beverages every year. Another problem is drug resistance which is responsible for approximately 2 million illnesses and around 23000 dead every year. Nearly 400,000 Americans acquire antibiotic-resistant Salmonella or Campylobacter each year. The aim of this study was to evaluate the outbreak of salmonellosis and shigellosis along with their antibiotic susceptibility patterns in different provinces of Iran.

Methods: Over a period of 2 years from 2015 to 2016, a total of 1055 cases in 249 outbreaks reported in 20 provinces of Iran, as a part of surveillance by the National Institute of Health (NIH). The stool samples of patients were taken and tested for Salmonella spp. and Shigella spp. by conventional standard techniques. Disk diffusion was used for the antibiotic sensitivity test.

Results: Of 1055 cases, $118(11.2 \%)$ contained Shigella and 74 (7\%) contained Salmonella. Antibiotic susceptibility tests showed that entirely $100 \%$ of Salmonella and Shigella isolates were susceptible to ciprofloxacin; whereas $12.2 \%$ of Salmonella and $98.2 \%$ of Shigella were resistant to cotrimoxazole.

Conclusion: Our results show that there is a need for more food handling practices to minimize the exposure of consumers to Salmonella and Shigella, at all points along the distribution chain.

Keywords: Foodborne, Outbreaks, Salmonellosis, Shigellosis

Conflicts of Interest: None declared

Funding: This paper is part of a research project approved by the Food Microbiology Research Center, Tehran University of Medical Sciences and Health Services Contract No. 20345.

*This work has been published under CC BY-NC-SA 1.0 license.

Copyright $@$ Iran University of Medical Sciences

Cite this article as: Soltan Dallal MM, Motalebi S, Masoumi Asl H, Sharifi Yazdi MK, Rahimi Forushani A. Antimicrobial investigation on the multi-state outbreak of salmonellosis and shigellosis in Iran. Med J Islam Repub Iran. 2020 (18 May);34:49. https://doi.org/10.47176/mjiri.34.49

\section{Introduction}

Foodborne illness outbreaks are defined when two or more people get same illness from same contaminated food. Foodborne illnesses are important public health concerns despite the fact that all effort has been made to pre- vent it $(1,2)$. Foodborne diseases are caused by the indigestion of contaminated food. In some cases they may result in either hospitalization or death. Nearly 250 etiologic agents of foodborne illnesses had been recognized so
Corresponding author: Dr Mohammad Mehdi Soltan Dallal, msoltanda@sina.tums.ac.ir

1. Food Microbiology Research Center, Tehran University of Medical Sciences, Tehran, Iran

2. Department of Food Microbiology, School of Public Medical Science, Tehran University Medical Science, Tehran, Iran

3. Center for Communicable Disease Control, Ministry of Health and Medical Education, Tehran, Iran

4. Zoonosis Research Centre, Tehran University of Medical Sciences, Tehran, Iran

5. Department of Medical Laboratory Sciences, School of Para Medicine, Tehran University of Medical Sciences, Tehran, Iran

6. Department of Epidemiology and Biostatics, School of Public Health, Tehran University of Medical Sciences, Tehran, Iran $\uparrow$ What is “already known” in this topic:

Salmonella spp. and Shigella spp. are the two most important bacteria causing foodborne diseases. Therefore special attention should be paid to these bacteria in any foodborne outbreak.

$\rightarrow$ What this article adds:

The finding of this research could be used for the epidemiological purpose in Iran. 
far (3). Most people infected with Salmonella develop symptoms such as diarrhea, fever, and abdominal cramps between 12 to 72 hours after digestion of contaminated food. The illness is self-limited and recovery takes between 4 to 7 days. But in some cases, diarrhea may be severe and needs hospitalization (4). Out of approximately 5.2 million cases of bacterial diarrhea that occur each year in the United States, $80 \%$ is related to foodborne illnesses (5). It is estimated that bacterial enteropathogens are responsible for 76 million illnesses, including 325,000 hospitalizations, and 5,000 deaths in the United States each year (6). The four most commonly reported bacterial enteropathogens in the United States are Campylobacter; non-typhoid Salmonella, Shiga toxin-producing $E$. coli, and Shigella (7). According to data from Centers for Disease Control and Prevention, infections with the following bacterial pathogens were detected in descending order of rates per 100,000 people in the United States in 2012: Salmonella, 16.4 cases; Campylobacter, 14.3 cases; Shiga toxin-producing Escherichia coli O157:H7 strain, 1.1 cases; Vibrio, 0.4 cases; and Yersinia, 0.3 cases. The rate of Shigella infection in the United States was 2.3 cases per 100,000 in 2011 (8). Salmonellosis outbreaks can occur in a wide range of foods such as fresh vegetables, fruits, meats, puffed cereal snacks, pre-packaged pot pies, and peanut (9).

Shigellosis is usually transmitted from person to person, but in some cases, the main cause of the disease is contaminated food such as water, milk, vegetables, and ice cream $(10,11)$. Signs and symptoms of food-borne diseases are abdominal cramps, nausea, vomiting, diarrhea, fever, and headache, depending on the severity of the disease. Symptoms usually appear between 24 to 48 hours, and stay for one or two days (11). Food-borne diseases can be dangerous in children, neonatal pregnant women, their fetuses, and persons with weakened immune systems $(12,13)$. A study carried out by Masoumi Asl et al. in Iran indicated that the outbreak rate has increased from $0.07 / 100000$ to $1.38 / 100000$ population during 2006 and rates of outbreaks in three provinces of Khuzestan, Kermanshah and Qazvin were more than expected (10). The use of antimicrobial agents in any environment might result in the survival of antibiotic-resistant pathogens (14). The use of antimicrobial agents to domestic livestock for the growth, prevention and treatment of diseases is an important factor in the emergence of antibiotic-resistant pathogens that subsequently transfer to humans through the food chain $(15,16)$. Nerveless most of antimicrobialresistant Salmonella are acquired by eating contaminated foods of animal origin $(17,18)$. This research was conducted to evaluate the surveillance of the multi-state outbreak of salmonellosis and shigellosis in Iran.

\section{Methods \\ Laboratory Investigation}

During the 2-year period from 2015 to 2016, a total of 1055 cases in 249 outbreaks reported in twenty providences of Iran, as a part of surveillance conducted by the Ministry of Health and Medical Education in Iran (10).

The outbreak samples were collected from health cen- ters during the outbreaks and were sent to us, as we were the collaborator laboratory of the Ministry of Health and Medical Education.

Stool samples of patients with the following symptoms, diarrhea, fever, abdominal pain, vomiting, and nausea were collected from different providences of Iran and tested in the laboratory of the department of public health for isolation and identification of Salmonella and Shigella by means of standard techniques and API 20E kit. For isolation of Salmonella, samples were inoculated in Selenite$\mathrm{F}$ broth at $37^{\circ} \mathrm{C}$ for 12 to 18 hours and for Shigella directly were streaked onto Hektoen enteric agar (Merck, Hamburg, Germany)-and incubated at $37^{\circ} \mathrm{C}$ for 24 hours. The suspicious gram-negative and oxidase-negative colonies were selected for identification using conventional biochemical tests, such as TSI, SIM, ODC, LIA, Simmons Citrate and Urea. The phenotypic biochemical profiles of isolates were compared with WHO criteria for Salmonella and Shigella (12).

\section{Susceptibility Testing}

Disc diffusion kit manufactured by MAST Company (Bootle, Merseyside, UK) was used for the susceptibility test according to guidelines by Clinical and Laboratory Standards Institute (19). A colony of the bacteria was inoculated in a test tube containing broth media. Broth culture was incubated at $35{ }^{\circ} \mathrm{C}$ until it reached $0.5 \mathrm{McF}$ arland turbidity. Broth was cultured on Mueller-Hilton agar plates (Merck) and antimicrobial disks were placed on the plates and incubated at $37^{\circ} \mathrm{C}$ for $16-18$ hours. Antimicrobial disks were used including: ampicillin $(10 \mathrm{mcg})$, amikacin (30 cg), chloramphenicol (30 mcg), cefotaxim (30 $\mathrm{mcg})$, gentamicin $(10 \mathrm{mcg})$, nalidixic acid $(30 \mathrm{mcg})$, cotrimoxazole $(10 \mathrm{mcg})$, tetracycline $(30 \mathrm{mcg})$, cephalexin (30 mcg), ciprofloxacin (5 mcg), imipenem (10 mcg), and ceftriaxon $(30 \mathrm{mcg})$, cefixim $(30 \mathrm{mcg})$, and clindamycin (30 mcg).

\section{Ethics}

This research was approved by the Ethics Committee of Tehran University of Medical Sciences (TUMS) (project number: 91-04-172-20345).

\section{Statistical Analysis}

Statistical analysis of results was performed using SPSS/PC 11.5 software (SPSS, Chicago, IL). Chi-square and Fisher's exact two-tailed tests were used. A P-value less than 0.05 was considered as statistically significant.

\section{Results}

Among the 1055 persons infected in 20 provinces, 249 $(23.6 \%)$ were hospitalized and $18(1.71 \%)$ were died. The percentage of Shigella and Salmonella isolates was $118(11.2 \%)$ and $74(7 \%)$ respectively. The results of isolation of Salmonella spp. and Shigella spp. are shown in Table 1. Sources of contamination were food (36.1\%), water $(9.7 \%)$, and uncertain sources $(54.2 \%)$. The demographic results (sex, age, job, and seasonal) are shown in Table 2.

Salmonellosis occurred mostly in spring (67.6\%) and 
Table 1. Percent frequency of Salmonella and Shigella species isolated from outbreaks

\begin{tabular}{lcc}
\hline Bacteria & Frequency & $\%$ \\
\hline Salmonella serogroup A & 8 & 10.8 \\
Salmonella serogroup B & 5 & 6.8 \\
Salmonella serogroup C & 51 & 68.9 \\
Salmonella serogroup D & 10 & 13.5 \\
Shigella sonnei & 105 & 89.0 \\
Shigella flexneri & 13 & 11.0 \\
Shigella dysenteriae & 0 & 0.0 \\
Shigella boydii & 0 & 0.0 \\
\hline
\end{tabular}

Table 2. Demographic distribution in outbreaks of Salmonella and Shigella

\begin{tabular}{lcc}
\hline Demographic information & Level & $\%$ \\
\hline Sex & Male & 61.5 \\
& Female & 38.5 \\
Age & $<1$ & 1.6 \\
& $1-7$ & 6.2 \\
& $7-25$ & 56.8 \\
& $25-65$ & 29.2 \\
Season & $>65$ & 6.2 \\
& Spring & 24.0 \\
& Summer & 42.2 \\
Location & Autumn & 21.8 \\
& Winter & 12.0 \\
& Urban & 78.1 \\
& Rural & 21.9 \\
\hline
\end{tabular}

Table 3. Results of analyzed outbreak of Salmonella $(\mathrm{n}=74)$ and Shigella $(\mathrm{n}=118)$ antimicrobial susceptibility testing, Iran

\begin{tabular}{lcccc}
\hline Antibiotic & $\begin{array}{c}\text { Salmonella } \\
\text { No. }\end{array}$ & $\%$ & $\begin{array}{c}\text { Shigella } \\
\text { No. }\end{array}$ & $\%$ \\
\hline Ciprofloxacin & 74 & 100 & 118 & 100 \\
Imipenem & 70 & 94.6 & - & - \\
Amikacin & 66 & 89.2 & 74 & 62.7 \\
Cloramphenicol & 64 & 86.5 & - & - \\
Cotrimoxazol & 59 & 79.7 & - & - \\
Ampicilin & 56 & 75.7 & 65 & 55.1 \\
Ceftriaxon & 56 & 75.7 & 107 & 90.7 \\
Cefotaxim & 46 & 62.2 & 100 & 84.7 \\
Tetracyclin & 31 & 41.9 & 18 & 15.3 \\
Cephalexin & 19 & 25.7 & 96 & 81.4 \\
Gentamaycin & 13 & 17.6 & 90 & 76.3 \\
Nalidixic Acid & 9 & 12.2 & 82 & 69.5 \\
Cefixim & - & - & 112 & 94.9 \\
Clindamycin & - & - & 19 & 16.1 \\
\hline
\end{tabular}

Shigellosis in summer $(50.8 \%)$. Male patients were dominated $(57.3 \%)$. Statistical analysis of K2 (chi-square) test indicates that the relation between risk of outbreak by water, food, season in which outbreak occurred, and the gender are significant $(\mathrm{p}<0.001)$. Most salmonellosis (70.3\%) occurred in Hamadan and shigellosis (54.2\%) occurred in Yazd province respectively. The outbreaks were happened in schools, restaurants, workplaces, domestic houses, dormitories, barracks, nursing houses, nurseries. All strains of Salmonella and Shigella were susceptible to ciprofloxacin, whereas $88 \%$ of Samonella and $30.5 \%$ of Shigella were resistant to nalidixic acid. The antibiotic resistance results are shown in Table 3.

\section{Discussion}

There are many reasons for the spread of food-borne diseases including increased number of travels and tourism, eating outside home, and change of eating habits (4, 20). Salmonella is a common cause of foodborne disease and is responsible for nearly $18 \%$ of outbreaks (21). Previous investigators have demonstrated that Salmonella was the cause of about $50 \%$ of food-borne diseases, followed by pathogenic Escherichia coli (33\%), Shigella (17\%) (22), and some other isolates of Salmonella (2.9\%) (23). Variety of foods such as peanut butter, leafy vegetables, sprouts, grains/beans, fruits/nuts, fungi, dairy, eggs, fish, pork, beef and chicken could be contaminated with Salmonellas and cause salmonellosis $(9,11,24,25)$. In this study, the percentage of Salmonella isolate was $11 \%$. Among the four isolated serotypes of Salmonella, serotype C $(68.9 \%)$ was the most common and serotype B $(6.8 \%)$ the least common serotypes. The highest incidence of outbreaks was observed during spring $(67.6 \%)$ and the lowest number in winter; this might be due to the higher consumption of fruits and vegetables in winter. Between the sources of contamination, $36.1 \%$ of Salmonella was isolated from foods, which is alarming. Shigellosis can spread easily in childcare facilities, and homeless shelters; and the outbreaks of shigellosis are difficult to control due to the ease of person-to-person transmission among young children (8). The results of this study suggest that effective control including participation in the intervention, supervised hand washing for children in schools, childcare centers, and homeless shelters can prevent the transmission of shigellosis into the community $(26,27)$. The most affected age ranges between 7 to 25 years $(61 \%)$ and the lowest was over 65 years $(6 \%)$. Male gender was dominated (male $61.8 \%$ and female $38.2 \%$ ). The percentage of outbreaks in the urban areas $(78 \%)$ compared to rural areas $(22 \%)$ indicates that the risk of outbreaks can be reduced by using proper hygiene. All of the isolated Salmonella and Shigella were sensitive to ciprofloxacin. In contrast to our results, studies conducted in Turkey showed a reduction in sensitivity to ciprofloxacin $(28,29)$. The result of this study showed that some of the isolates Shigella spp were resistance to ampicilin which is commonly used for the treatment of Shigella infections. Various studies have reported an increase in antimicrobial resistance amongst different species of Shigella spp. (30). Based on our findings, the most commonly observed resistance of Shigella strains were to tetracycline $(84.7 \%)$, ampicillin $(44.9 \%)$ and clindamycin $(83.9 \%)$ and lowest resistant was found to ciprofloxacin (100\%). However, a previous study reported the highest resistance rates $(61.8 \%)$ to tetracycline (25). The role of antimicrobials in the control of epidemic shigellosis are not a substitute for hygienic measures in reducing the secondary spread of shigellosis. Antimicrobials should be reserved for treatment of patients only when clinically indicated, to prevent the resistant strains of Shigella $(30,31)$. Prophylactic cannot be recommended to prevent illness in persons who are exposed but not ill. Furthermore using antimicrobials to treat patients with mild shigellosis to reduce the spread of secondary infections is not more effective in preventing Shigella infections than hand washing with soap and water $(27,32)$. Since resistance patterns may change, antimicrobial selection should be based on antimicrobial resistance results of strains. Shigellosis outbreaks are more common in the summertime $(33,34)$. Most Salmonella infections in 
humans result from the ingestion of contaminated food such as poultry, beef, pork, eggs and milk (11, 24, 25). Intestinal Salmonellosis is self-limited and recovery will be obtained in several days without antibiotics. When the infection spreads beyond the intestinal tract, antibiotics such as ciprofloxacin in adults and ceftriaxone in children could be used for the treatment. Since antimicrobial agents are given to domestic livestock for the prevention and treatment of disease, antimicrobial-resistant Salmonella could transfer from contaminated foods of animal origin $(11,27,33)$. All stains of Salmonella were susceptible to ciprofloxacin, imipenem (94.6\%), amikiacin (89.2\%), and only $(12.2 \%)$ to nalidixic acid. In another study, all Salmonella isolates were susceptible to nalidixic acid (35), compared to our results $(12.2 \%)$. These findings demonstrate the multidrug-resistance strains of Salmonella including nalidixic acid resistant isolates (23). The results of this study indicate that proper strategy is necessary to reduce the prevalence of antimicrobial resistant Salmonella in food by means of proper guidelines for the use of antibacterial agents in animal foods.

\section{Conclusion}

Our two-year period analysis of food poisoning cases, illustrated that Salmonella and Shigella species were the most common causative agent of food poisoning. Therefore proper food safety instruction should apply for either food manufacturer or suppliers in order to prevent foodborne disease outbreaks.

\section{Conflict of Interests}

The authors declare that they have no competing interests.

\section{References}

1. WHO (2012) Reducing foodborne diseases by educating consumers. Geneva: World Health Organization. Available at: http://www. who.int/foodsafety/en/.Accessed 15 December 2013.

2. Scallan E, Hoekstra RM, Angulo FJ, Tauxe RV, Widdowson MA, Roy SL, et al. Foodborne illness acquired in the United States major pathogens. Emerg Infect Dis. 2011;17:7-15.

3. Linscott AJ. Food-Borne Illnesses. Clinical Microbiology Newsletter, 2011;33:41-45.

4. Centers Disease for control and prevention. CDC, Infection with Salmonella, Salmonella in the Caribbean 2013.

5. Painter JA, Hoekstra RM, Ayers T, Tauxe RV, Braden CR, Angulo FJ, et al. Attribution of foodborne illnesses, hospitalizations, and deaths to food commodities by using outbreak data, United States, 1998-2008. Emerg Infect Dis. 2013 Mar;19(3):407-15.

6. Centers for Disease Control and Prevention (CDC). Vital signs: incidence and trends of infection with pathogens transmitted commonly through food--foodborne diseases active surveillance network, 10 U.S. sites, 1996-2010. MMWR Morb Mortal Wkly Rep. 2011 Jun 10;60(22):749-55.

7. Nyachuba DG. Foodborne illness: is it on the rise? Nutr Rev. 2010 May;68(5):257-69.

8. National enteric disease surveillance: Shigella annual report, 2011. Atlanta: Centers for Disease Control and Prevention, 2013.

9. Pires SM, Vieira AR, Hald T, Cole D. Source attribution of human salmonellosis: an overview of methods and estimates. Foodborne Pathog Dis. 2014 Sep;11(9):667-76.

10. Masoumi Asl H, Gouya MM, Soltan Dallal MM, Aghili N. Surveillance for foodborne disease outbreaks in Iran,2006-2011. Med J Islam Repub Iran. 2015;29:285.

11.Gould LH, Walsh KA, Vieira AR, Herman K, Williams IT, Hall AJ, et al. Surveillance for foodborne disease outbreaks - United States,
1998-2008. MMWR Surveill Summ. 2013 Jun 28;62(2):1-34.

12. Soltan Dallal MM, Motalebi S, Masoumi Asl H, Rahimi Forushani A, Sharifi Yazdi MK, Rajabi Z, et al. Analysis of epidemiological data of foodborne outbreak reported in Iran. Tehran Uni Med J. February 2015;72(11):780-788.

13. Broner S, Torner N, Dominguez A, Martinez A, Godoy P. Sociodemographic inequalities and outbreak of foodborne disease. an ecologic study. Food Control. 2010;21(6):947 -51.

14. Elal Mus T, Cetinkaya F, Cibik R, Soyutemiz GE, Simsek H, Coplu N. Pathogenicity determinants and antibiotic resistance profiles of enterococci from foods of animal origin in Turkey. Acta Vet Hung. 2017 Dec;65(4):461-474.

15. Hamed OM, Sabry MA, Hassanain NA, Hamza E, Hegazi AG, Salman MB. Occurrence of virulent and antibiotic-resistant Shiga toxin-producing Escherichia coli in some food products and human stool in Egypt. Vet World. 2017 Oct;10(10):1233-1240.

16. Boireau C, Morignat É, Cazeau G, Jarrige N, Jouy É, Haenni M, et al. Antimicrobial resistance trends in Escherichia coli isolated from diseased food-producing animals in France: A 14-year period timeseries study. Zoonoses Public Health. 2018 Feb;65(1):e86-e94.

17. Wang Y, Cao C, Alali WQ, Cui S, Li F, Zhu J, et al. Distribution and antimicrobial susceptibility of foodborne Salmonella serovars in eight provinces in China from 2007 to 2012 (Except 2009). Foodborne Pathog Dis. 2017 Jul;14(7):393-399.

18. Thai TH, Hirai T, Lan NT, Yamaguchi R. Antibiotic resistance profiles of Salmonella serovars isolated from retail pork and chicken meat in North Vietnam. Int J Food Microbiol. 2012 May $15 ; 156(2): 147-51$

19. Centers for Disease Control and Prevention (CDC). Salmonella enteritidis infections associated with foods purchased from mobile lunch trucks - alberta, Canada, october 2010-february 2011. MMWR Morb Mortal Wkly Rep. 2013;62(28):567-9.

20. Broglia A, Kapel C. Changing dietry habits in a changing world: drivers for the transmission of foodborne parasitic zoonoses. Vet Parasitol. 2011 Nov 24;182(1):2-13.

21. Centers for Disease Control and Prevention (CDC). Surveillance for foodborne disease outbreaks -United States, 2007. MMWR Morb Mortal Wkly Rep. 2010 Aug 13;59(31):973-9.

22. Kozak GK, MacDonald D, Landry L, Farber JM. Foodborne outbreaks in Canada linked to produce: 2001 through 2009. J Food Prot. 2013;76:173-83.

23. Soltan Dallal MM, Khalilian M, Masoumi Asl H, Bakhtiari R, Davoodabadi A, Rajabi Z. Identification of Salmonella serotypes in foodborne outbreaks by sequencing of ITS region of 16S-23SrRNA. J Shahrekord Uni Med Sci. 2016;18(1):73-80.

24. Hanning IB, Nutt JD, Ricke SC. Salmonellosis outbreaks in the United States due to fresh produce: sources and potential intervention measures. Foodborne Pathog Dis. 2009 Jul-Aug;6(6):635-48.

25. Soltan Dallal MM, TaremiM, GachkarL, Modarressi S, Sanaei M, Bakhtiari R, et al. Characterization of antibiotic resistant patterns of Salmonella serotypes isolated from meat and chicken samples in Tehran. Jundishapur J Microb. 2009;2(4):124- 31.

26. Ejemot-Nwadiaro RI, Ehiri JE, Arikpo D, meremikwu MM, Critchley. Hand washing promotion for preventing diarrhoea. Cochrane Database Syst Rev. 2015 Sep 3;(9):CD004265.

27. Debas G, Kibret M, Biadglegne F, Abera B. Prevalence and antimicrobial susceptibility patterns of Shigella species at Felege Hiwot Referral Hospital, Northwest Ethiopia. Ethiop Med J. 2011 Jul;49(3):249-56.

28. Johnson NB, Hayes LD, Brown K, Hoo EC, Ethier KA; Centers for Disease Control and Prevention (CDC). CDC National Health Report: leading causes of morbidity and mortality and associated behavioral risk and protective factors--United States, 2005-2013. MMWR Suppl. 2014 Oct 31;63(4):3-27.

29. Lindgren MM, Kotilainen P, Huovinen P, Hurme S, Lukinmaa $\mathrm{S}$, Webber MA, et al. Reduced fluoroquinolone susceptibility in Salmonella enterica isolates from travelers, Finland. Emerg Infect Dis. 2009 May; 15(5):809-12.

30. Khan E, Jabeen K, Ejaz M, Siddiqui J, Shezad MF, Zafar A. Trends in antimicrobial resistance in Shigella species in Karachi, Pakistan. J Infect Dev Ctries. 2009 Dec 15;3(10):798-802.

31. Debnath F, Mukhopadhyay AK, Chowdhury G, Saha RN, Dutta S. An Outbreak of Foodborne Infection Caused by Shigella sonnei in West Bengal, India. Jpn J Infect Dis. 2018 Mar 22;71(2):162-166.

32. Mosquito S, Zegarra G, Villanueva C, Ruiz J, Ochoa TJ. Effect of 
bovine lactoferrin on the minimum inhibitory concentrations of ampicillin and trimethoprim-sulfamethoxazole for clinical Shigella spp. strains. Biochem Cell Biol. 2012 Jun;90(3):412-6

33. Nygren BL, Schilling KA, Blanton EM, Silk BJ, Cole DJ, Mintz ED. Foodborne outbreaks of shigellosis in the USA, 1998-2008. Epidemiol Infect. $2013 \mathrm{Feb}$;141(2):233-41.

34. Soltan Dallal MM, Motalebi S, Masoomi Asl H, Rahimi Forushani A, Kalantar E. Burden of food-related illness caused by resistant Salmonella spp. and Shigella spp.: harbingers of multistate outbreaks in 2012 and 2013. Int J Enteric Pathog. 2015 November;3(4):e29276.

35. Lindgren MM, Kotilainen P, Huovinen P, Hurme S, Lukinmaa $\mathrm{S}$, Webber MA, et al. Reduced fluoroquinolone susceptibility in Salmonella enterica isolates from travelers, Finland. Emerg Infect Dis. 2009 May; 15(5):809-12. 